\title{
O Uso da Captura de Movimentos no Desenvolvimento de Produtos: um estudo focado nas tecnologias e aplicações
}

\author{
The Use of Motion Capture in Product Development: a study focused on technologies \\ and applications
}

\author{
VARNIER, Thiago; Mestrando em Design; Universidade Federal de Santa Catarina \\ thiagovarnier1@gmail.com \\ MERINO, Eugenio Andrés Díaz, Doutor em Eng. Produção; Universidade Federal de Santa Catarina \\ eugenio.merino@ufsc.br
}

\begin{abstract}
Resumo
A ergonomia visa a adequação entre produto e usuário, considerando suas capacidades e limitações físicas no desenvolvimento de projetos. Nesta perspectiva, este artigo pretende identificar as tecnologias utilizadas para a captura de movimentos e suas aplicações no desenvolvimento de produtos por meio de uma revisão sistemática. Foram encontrados 221 documentos, e após minerálos, realizou-se análises bibliométricas e de conteúdo. Identificou-se os sistemas Óptico, Kinect e Inercial. No que tange as aplicações, destaca-se sua utilização para aprimorar o processo de desenvolvimento de projetos, apresentando informações objetivas dos movimentos de segmentos e articulações corporais. Ainda, contribui para a concepção de novos produtos guiando as etapas do processo, e auxilia na avaliação e análise dos produtos. Contudo, percebe-se que a captura de movimentos pode ser uma ferramenta eficiente para obtenção de dados quantitativos na área de design, gerando informações e definições precisas da biomecânica, aproximando o projetista as necessidades reais do usuário.
\end{abstract}

Palavras Chave: Design; Ergonomia; Captura de Movimentos.

\begin{abstract}
Ergonomics aims at the adequacy between product and user, considering their capacities and physical limitations in the development of projects. In this perspective, this article intends to identify the technologies used for the capture of movements and its applications in the development of products through a systematic review. A total of 221 documents were found, and after mining, bibliometric and content analyzes were performed. Optical, Kinect and Inertial systems were identified. Regarding the applications, it stands out its use to improve the process of development of projects, presenting objective information of the movements of segments and body articulations. Also, it contributes to the design of new products guiding the process steps, and assists in the evaluation and analysis of the products. However, it can be seen that motion capture can be an efficient tool to obtain quantitative data in the design area, generating accurate information and definitions of biomechanics, bringing the designer closer to the user's real needs.
\end{abstract}

Keywords: Design; Ergonomics; Motion Capture. 


\section{Introdução}

Bonsiepe (2012) afirma que o Design integra ciência e tecnologia, com foco na intersecção entre usuário e produto. Assim, a preocupação em projetar levando-se em conta o usuário, por meio desse enfoque integrador, faz com que o Design seja um campo híbrido, que opera entre corpo e informação, entre artefato, usuário e sistema (CARDOSO, 2012). Neste sentido, Best (2015) corrobora que o Design é um processo de resolução de problemas, e uma atividade centrada nas pessoas, não se limitando ao projeto de objetos ou adição de estética (MARTINS, MERINO, 2011; BONSIEPE, 2012).

A este respeito Cambiaghi (2012) ressalta a necessidade de levar em conta as dimensões dos indivíduos para o projeto de produtos únicos, reguláveis ou mesmo uma gama de produtos capazes de atender a todos. Dessa forma, a autora salienta que a compreensão das medidas das várias partes do corpo humano possibilita atender melhor às necessidades funcionais dos usuários para o desenvolvimento de projetos. Ainda, Oliveira (2011) complementa que adequar os produtos aos usuários de maneira a considerar suas capacidades e limitações requer o conhecimento dos movimentos corporais.

Neste particular, uma das áreas que preocupa-se com a compreensão das interações entre seres humanos e outros elementos de um sistema é a Ergonomia. Segundo a IEA (2017) a ergonomia, tem por objetivo adequar as ferramentas e ambientes de trabalho, com a finalidade de alcançar a melhor integração entre o produto e seus usuários, no contexto do desempenho da tarefa, abrangendo os aspectos físicos, cognitivos e organizacionais. Dessa forma, a ergonomia em sua abrangência física, foco desta pesquisa, está relacionada com as características da anatomia humana, antropometria, fisiologia e biomecânica, o que contribui para a concepção e avaliação de tarefas, produtos, ambientes e sistemas afim de torná-los compatíveis com as necessidades, capacidades e limitações dos usuários (IEA, 2017). Logo, é possível perceber, que os aspectos físicos complementam, ou devem ser complementados pelos aspectos cognitivos e organizacionais possibilitando uma compreensão global da situação.

Diante desse cenário, Merino et al. (2016 a, b) destacam a importância de incorporar nas pesquisas de Design instrumentos tecnológicos para a obtenção de dados objetivos referentes às capacidades físicas do usuário, que venham a identificar as sobrecargas biomecânicas, e auxiliar na aproximação do projetista às necessidades reais do usuário. Neste sentido Bittencourt (2011) salienta que a antropometria é uma área de estudo que aplica métodos científicos de medidas físicas nos seres humanos com o objetivo de se obter informações precisas e realistas utilizáveis no desenvolvimento de projetos, buscando adequar os produtos a cada usuário.

Não obstante, Neumann (2011) corrobora que as informações antropométricas são valiosas para os projetos de desenvolvimento de ambientes de trabalho, mobiliário, ferramentas, equipamentos, entre outros, e justifica que existem muitos métodos para medir objetivamente 0 movimento humano, incluindo eletrogoniometria, acelerometria, técnicas de imagem, dispositivos de monitoramento eletromagnético e sistemas de captura de movimentos. Dessa forma, pode-se dizer que estes métodos permitem a obtenção de dados objetivos e precisos sobre o movimento humano, o que possibilita a compreensão dos aspectos essenciais do problema e permite $o$ desenvolvimento de projetos mais eficientes.

Mediante o contexto apresentado, este artigo tem como objetivo identificar as tecnologias utilizadas para a captura de movimentos e suas aplicações no desenvolvimento de produtos por 
meio de uma busca sistemática nos bancos de teses e dissertações (Repositório da UFSC, Banco de Teses da Capes, Biblioteca Digital Brasileira de Teses e Dissertações - BDTD e a Dissertations \& Theses - ProQuest), bem como nas bases de dados (Scopus, Web of Science, PubMed e Scielo). Com isso, buscou-se responder a seguinte questão: Como a captura de movimentos vem sendo aplicada no desenvolvimento de produtos e/ou produtos de design e quais os tipos de equipamentos tecnológicos mais utilizados? Para tanto, serão enquadrados nesta pesquisa, os estudos que utilizaram a captura de movimentos como forma de auxiliar ou guiar no processo de desenvolvimento de produtos.

\section{Procedimentos Metodológicos}

A pesquisa possui natureza pura, por tratar de uma pesquisa que visa a ampliação de conhecimentos teóricos (MARCONI; LAKATOS, 2007), com objetivo exploratório e descritivo. O estudo exploratório é particularmente útil quando se dispõe de poucas informações (HAIR et.al, 2005). Por sua vez, os estudos descritivos buscam especificar as propriedades e as características do fenômeno que se submeta a uma análise (SAMPIERI; COLLADO; LUCIO, 2013). Dessa forma, esta pesquisa propôs o levantamento e análise da utilização da captura de movimentos no desenvolvimento de produtos.

Quanto aos procedimentos técnicos, classifica-se como estudo bibliográfico, pois analisa estudos já publicados sobre o assunto. A abordagem será mista, compreendendo a análise quantitativa e qualitativa dos dados oriundos da revisão sistemática (CRESWELL, 2014). A análise quantitativa compreendeu a análise bibliométrica do número de duplicatas, número de publicações por ano, países de ocorrência, os principais grupos de pesquisas e Instituições de Ensino Superior envolvidas, bem como as áreas de concentração e o fator de impacto dos periódicos, enquanto a análise qualitativa compreendeu a construção da matriz de síntese do conteúdo, referente a abordagem dos temas apresentados nos documentos, como também o tipo de equipamento tecnológico utilizado para a captura de movimentos, selecionados por meio da leitura dos artigos na íntegra.

\subsection{Etapas da Pesquisa}

De acordo com o objetivo dessa pesquisa, foi realizada uma revisão sistemática, utilizando o método proposto por Sampaio e Mancini (2007), que descrevem o desenvolvimento da revisão sistemática em cinco etapas: (I) Definir a pergunta; (II) Buscar a evidência; (III) Revisar e selecionar os estudos; (IV) Analisar a qualidade metodológica dos estudos; e (V) Apresentar os resultados.

\subsection{Materiais e Métodos}

Seguindo o método proposto por Sampaio e Mancini (2007), foi definida a seguinte pergunta de pesquisa: Como a captura de movimentos vem sendo aplicada no desenvolvimento de produtos e/ou produtos de design e quais os tipos de equipamentos tecnológicos mais utilizados?

Para tanto, a busca pelas evidências se deu em estudos nacionais e internacionais encontrados nos bancos de Teses e Dissertações Nacionais (Repositório da UFSC, Banco de Teses da Capes, Biblioteca Digital Brasileira de Teses e Dissertações - BDTD) e Internacionais (Dissertations \& Theses - ProQuest). Essas bases foram selecionadas seguindo os seguintes critérios: partiu-se do âmbito local (Florianópolis/SC) ao global (mundo). Primeiramente, foi realizada uma pesquisa no Repositório da UFSC, seguido do Banco de teses da CAPES, que reúne os resumos de teses e dissertações defendidas nos programas de pós-graduação do Brasil desde 1987 (CATÁLAGO DE 
TESES E DISSERTAÇÕES, 2018). Posteriormente buscou-se na Biblioteca Digital Brasileira de Teses de Dissertações do Instituto Brasileiro de Informação em Ciência e Tecnologia, que integra os sistemas de informações sobre teses e dissertações existentes nas instituições brasileiras (BDTD, 2018). Por fim, fez-se o mesmo levantamento na base de dados Banco de Dissertations \& Theses, banco de teses e dissertações disponíveis na íntegra do mundo (PROQUEST, 2018).

A pesquisa também ocorreu nas bases de periódicos da Scopus, por ser a maior base de dados de resumos e citações da literatura acadêmica, contendo mais de 15 mil periódicos indexados (SCOPUS, 2018); da Web of Science, por possuir mais de 9 mil periódicos indexados, os quais são os mais citados de suas respectivas áreas, e por abranger as áreas das ciências da saúde e das ciências sociais e aplicadas (WEB OF SCIENCE, 2018), da PubMed, por indexar mais de 4,5 milhões de textos completos de literatura de periódicos de ciências biomédicas e de ciências da vida (PUBMED, 2018) e da Scielo, por ser a base de maior referência para publicações brasileiras, com 355 periódicos indexados e mais de 300 mil referências (SCIELO, 2018). Vale ressaltar que todas as bases foram acessadas através do sistema CAPES.

Foram analisados todos os documentos provenientes da seguinte string de busca: (("motion capture" OR "motion tracking") AND ("product developement" OR "product design")). Os critérios de inclusão para seleção dos documentos dos bancos de teses e dissertações foram: string no (texto completo). Já para as bases de periódicos foram: string no (título, resumo e palavras-chave; artigos e artigos de anais de congresso).

Não houve restrições quanto à área. Quanto à língua, foram buscados apenas referências escritas em idiomas dominados pelos autores (português, inglês e espanhol). As buscas foram limitadas por datas de publicações, selecionado o período de 2012 a 2017. Também foi definido que seriam excluídos da revisão artigos de reviews, bem como os artigos não encontrados nas plataformas Capes, Google, Google Scholar ou que não fossem fornecidos pelos autores.

Dessa forma, os documentos obtidos foram exportados para o software EndNote X6, e selecionados considerando sua relação com o tema pesquisado, baseados na análise do título, palavras-chave e resumo dos artigos encontrados. Após a exclusão dos artigos que não tinham relação com o tema, foi realizada a leitura dos artigos na íntegra, sendo que o critério de seleção dos trabalhos foi o nível de contribuição para responder a pergunta de pesquisa proposta.

Posteriormente para realizar a análise das publicações selecionadas e construir a matriz de síntese, utilizou-se o Microsoft Excel 2016 e suas funções. A síntese dos resultados foi feita mediante a construção de duas matriz de análise do conteúdo: (1) referente a análise bibliométrica e a (2) com os dados referente a abordagem dos documentos e o tipo de equipamento tecnológico utilizado para a captura de movimentos. Por fim, elaborou-se uma síntese visual dos resultados por meio de um infográfico.

\section{Resultados e discussão}

Os resultados da revisão sistemática foram separados em dois tópicos: análise quantitativa que compreende a análise bibliométrica e análise qualitativa que compreende a análise do conteúdo dos documentos selecionados.

\subsection{Análise Bibliométrica}

A pesquisa nas bases de dados de Teses e dissertações foi realizada no dia 01 de novembro 
de 2017 e retornou um o total de 137 documentos, sendo 02 retirado do Repositório da UFSC, 01 da BDTD, 134 da ProQuest e nenhum retorno do Banco de Teses da Capes. Minerando os documentos duplicados no filtro 1 (F1), 136 restaram para o filtro do título, resumo e palavras-chave (F2). Neste filtro foram descartados 132 documentos por não conterem relação com o assunto de pesquisa, ou seja, não utilizaram a captura de movimentos no desenvolvimento de produtos. Assim, com os 04 documentos restantes, fez-se a busca dos arquivos disponíveis para realização da leitura dinâmica dos documentos na íntegra. Após a realização da leitura dinâmica foram selecionados (03 teses e 1 dissertações). A figura 1 apresenta o processo de filtragem dos documentos.

Figura 1 - Processo de seleção dos documentos nos bancos de Teses e Dissertações

\section{Pesquisa Bases de Teses e Dissertações}

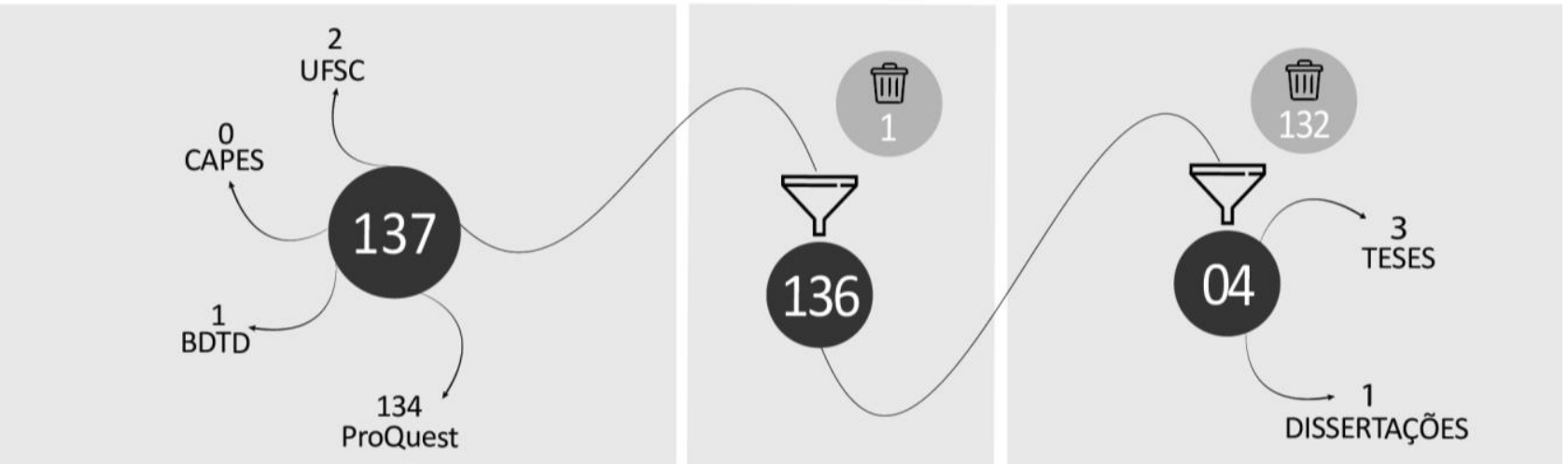

\section{F1 - Duplicados}

\section{F2-Título/Resumo/ Palavras- chave}

Fonte: elaborado pelos autores.

O quadro 1 apresenta o portfólio final dos 4 documentos selecionados nos bancos de teses e dissertações, sendo estes organizados pelo ano (do mais recente para o mais antigo). O quadro apresenta o autor, ano, Instituição de Ensino Superior, país, título do trabalho, o tipo de documento (tese ou dissertação) e a base de onde foi selecionado.

Quadro 1 - Portfólio final das Teses e Dissertações nacionais e internacionais

\begin{tabular}{|c|c|c|c|c|c|c|}
\hline AUTOR & ANO & IES & PAÍS & TÍTULO & TIPO & BASE \\
\hline JUN & 2015 & $\begin{array}{l}\text { State University } \\
\text { of New York at } \\
\text { Buffalo }\end{array}$ & $\begin{array}{l}\text { Estados } \\
\text { Unidos }\end{array}$ & $\begin{array}{l}\text { A home-based rehabilitation system } \\
\text { for deficient knee patients }\end{array}$ & Tese & ProQuest \\
\hline SOLOMON & 2015 & Tufts University & $\begin{array}{l}\text { Estados } \\
\text { Unidos }\end{array}$ & $\begin{array}{l}\text { Application of human factors } \\
\text { engineering in the redesign of an } \\
\text { oscillating bone saw to reduce high } \\
\text { risk postures of the wrist and to } \\
\text { enhance performance }\end{array}$ & Tese & ProQuest \\
\hline FERNANDES & 2014 & $\begin{array}{l}\text { Universidade } \\
\text { Federal } \\
\text { de Santa } \\
\text { Catarina }\end{array}$ & Brasil & $\begin{array}{l}\text { Ergonomia e Projeto: Contribuições } \\
\text { no projeto de ferramentas manuais } \\
\text { para agricultura familiar com ênfase } \\
\text { nos reflexos físicos da atividade de } \\
\text { extração manual de mandioca }\end{array}$ & Tese & $\begin{array}{l}\text { Repositório } \\
\text { UFSC }\end{array}$ \\
\hline
\end{tabular}




\begin{tabular}{llllll}
\hline SALVALAIO 2012 & $\begin{array}{l}\text { Universidade } \\
\text { Federal do Rio } \\
\text { Grande do Sul }\end{array}$ & Brasil & $\begin{array}{l}\text { Contribuição ao estudo da captura } \\
\text { do movimento aplicado ao design } \\
\text { em Tecnologia Assistiva }\end{array}$ & BDTD & \\
& & &
\end{tabular}

Fonte: elaborado pelos autores.

A pesquisa nas bases de dados também foi realizada no dia 01 de novembro de 2017 e retornou o total de 84 artigos, sendo 28 retirados da base de dados Scopus, 09 da Web of Science, 29 da PubMed e 18 da Scielo. Minerando os artigos duplicados no filtro (F1), 77 artigos restaram para o filtro do título, resumo e palavras-chave (F2). Neste filtro foram descartados 64 artigos por não conterem relação com o assunto de pesquisa proposto. Assim, com os 13 documentos restantes, fez-se a busca dos arquivos disponíveis para realização da leitura dinâmica dos documentos na íntegra. Após a realização da leitura dinâmica foram selecionados 13 artigos. A figura 2 apresenta o processo de filtragem dos documentos.

Figura 2 - Processo de seleção dos documentos nas bases de periódicos

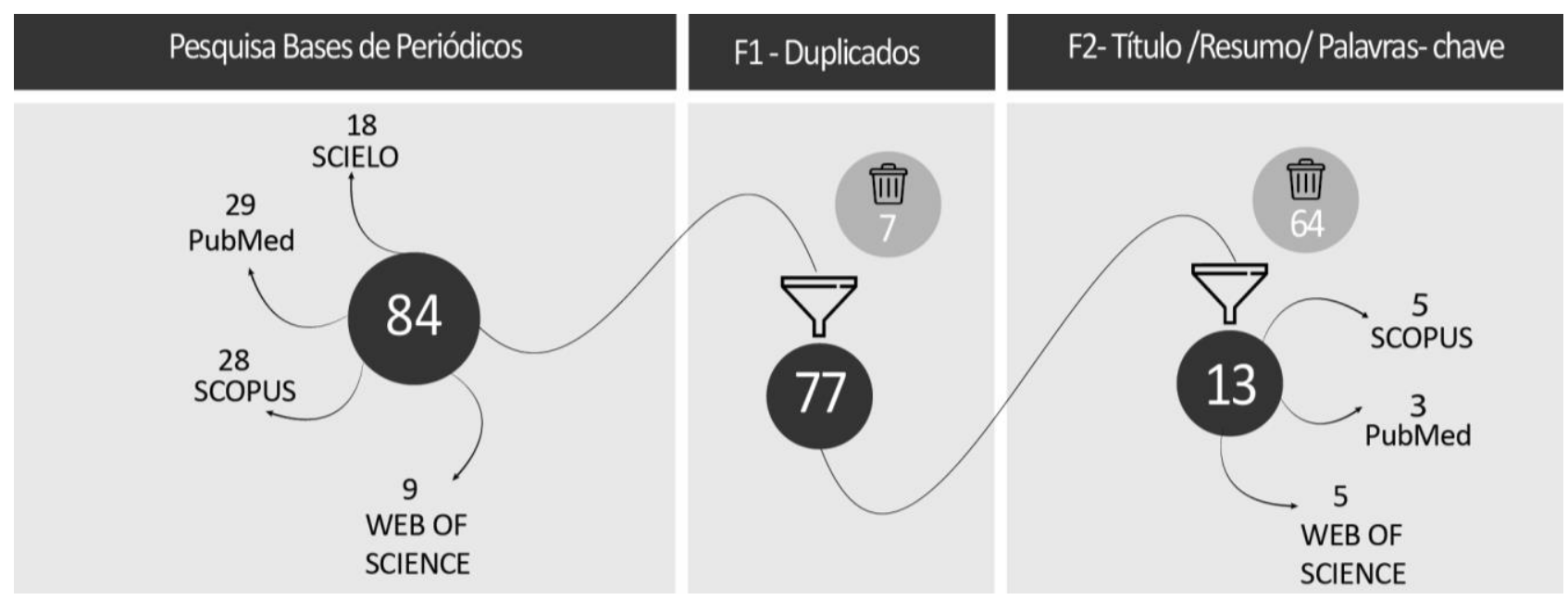

Fonte: elaborado pelos autores.

O quadro 2 apresenta o portfólio final dos 13 trabalhos selecionados nas bases de dados de periódicos, sendo estes organizados por ano (do mais recente para os mais antigo). O quadro apresenta os autores, ano, país, título do trabalho, o periódico/evento em que se encontra, o fator de impacto (JCR) do periódico e a base de onde foi selecionado.

Quadro 2 - Portfólio final dos 13 trabalhos selecionados nas bases de periódicos

\begin{tabular}{|c|c|c|c|c|c|c|}
\hline AUTOR & ANO & PAÍS & TÍTULO & $\begin{array}{l}\text { PERIÓDICO/ } \\
\text { EVENTO }\end{array}$ & JCR & BASE \\
\hline OYEKAN et al. & 2017 & $\begin{array}{l}\text { Reino } \\
\text { Unido }\end{array}$ & $\begin{array}{l}\text { Remote real-time collaboration } \\
\text { through synchronous exchange of } \\
\text { digitised human-workpiece } \\
\text { interactions }\end{array}$ & $\begin{array}{l}\text { Future Generation } \\
\text { Computer Systems }\end{array}$ & 3,997 & $\begin{array}{l}\text { Web of } \\
\text { Science }\end{array}$ \\
\hline $\begin{array}{l}\text { DEMIREL; } \\
\text { DUFFY }\end{array}$ & 2017 & $\begin{array}{l}\text { Estados } \\
\text { Unidos }\end{array}$ & $\begin{array}{l}\text { Incorporating Tactile Cues into } \\
\text { Human-Centered Virtual Product } \\
\text { Design }\end{array}$ & $\begin{array}{l}\text { Human Factors and } \\
\text { Ergonomics In } \\
\text { Manufacturing }\end{array}$ & 0,761 & $\begin{array}{l}\text { Web of } \\
\text { Science/ } \\
\text { Scopus }\end{array}$ \\
\hline
\end{tabular}




\begin{tabular}{|c|c|c|c|c|c|c|}
\hline LEE et al. & 2017 & $\begin{array}{l}\text { Coreia do } \\
\text { Sul }\end{array}$ & $\begin{array}{l}\text { A 3D Human-Machine Integrated } \\
\text { Design and Analysis Framework for } \\
\text { Squat Exercises with a Smith } \\
\text { Machine }\end{array}$ & Sensors & 2,677 & PubMed \\
\hline $\begin{array}{l}\text { JOUNG; LI; } \\
\mathrm{NOH}\end{array}$ & 2016 & $\begin{array}{l}\text { Coreia do } \\
\text { sul }\end{array}$ & $\begin{array}{l}\text { XML-based neutral schema for } \\
\text { automated ergonomic analysis with } \\
\text { digital human simulation and inline } \\
\text { motion capture }\end{array}$ & $\begin{array}{l}\text { International } \\
\text { Journal of } \\
\text { Computer } \\
\text { Integrated } \\
\text { Manufacturing } \\
\end{array}$ & $\mathrm{NE}$ & $\begin{array}{l}\text { Web of } \\
\text { Science/ } \\
\text { Scopus }\end{array}$ \\
\hline CHOI; LEE & 2015 & $\begin{array}{l}\text { Coreia do } \\
\text { Sul }\end{array}$ & $\begin{array}{l}\text { Discomfort Evaluation of Truck } \\
\text { Ingress/Egress Motions Based on } \\
\text { Biomechanical Analysis }\end{array}$ & Sensors & 2,677 & PubMed \\
\hline VELAZ et al. & 2014 & Espanha & $\begin{array}{l}\text { Natural and hybrid bimanual } \\
\text { interaction for virtual assembly } \\
\text { tasks }\end{array}$ & Virtual Reality & 0.628 & $\begin{array}{l}\text { Web of } \\
\text { Science/ } \\
\text { Scopus }\end{array}$ \\
\hline $\begin{array}{l}\text { ESPITIA- } \\
\text { CONTRERAS; } \\
\text { SANCHEZ- } \\
\text { CAIMAN; } \\
\text { URIBE- } \\
\text { QUEVEDO }\end{array}$ & 2014 & Colômbia & $\begin{array}{l}\text { Development of a Kinect-based } \\
\text { anthropometric } \\
\text { application }\end{array}$ & $\begin{array}{l}\text { Proceedings - IEEE } \\
\text { Virtual Reality }\end{array}$ & - & $\begin{array}{l}\text { Web of } \\
\text { Science/ } \\
\text { Scopus }\end{array}$ \\
\hline $\begin{array}{l}\text { JOUNG; } \\
\mathrm{NOH}\end{array}$ & 2014 & $\begin{array}{l}\text { Coreia do } \\
\text { Sul }\end{array}$ & $\begin{array}{l}\text { Integrated modeling and simulation } \\
\text { with in-line motion captures for } \\
\text { automated ergonomic analysis in } \\
\text { product lifecycle management }\end{array}$ & $\begin{array}{l}\text { Concurrent } \\
\text { Engineering } \\
\text { Research and } \\
\text { Applications }\end{array}$ & $\mathrm{NE}$ & $\begin{array}{l}\text { Web of } \\
\text { Science/ } \\
\text { Scopus }\end{array}$ \\
\hline JI; JUN & 2014 & $\begin{array}{l}\text { Coreia do } \\
\text { Sul }\end{array}$ & $\begin{array}{l}\text { Elementary implementation of a } \\
\text { parameter-based generative design } \\
\text { system considering the user } \\
\text { environment }\end{array}$ & $\begin{array}{l}\text { Journal of Asian } \\
\text { Architecture and } \\
\text { Building } \\
\text { Engineering }\end{array}$ & 0,277 & $\begin{array}{l}\text { Web of } \\
\text { Science/ } \\
\text { Scopus }\end{array}$ \\
\hline QING et al. & 2013 & $\begin{array}{l}\text { Reino } \\
\text { Unido }\end{array}$ & $\begin{array}{l}\text { Application of JACK on evaluation of } \\
\text { a split seat chair }\end{array}$ & $\begin{array}{l}\text { ICAC } 2013 \text { - } \\
\text { Proceedings of the } \\
\text { 19th International } \\
\text { Conference on } \\
\text { Automation and } \\
\text { Computing: Future } \\
\text { Energy and } \\
\text { Automation }\end{array}$ & - & Scopus \\
\hline MA et al. & 2013 & China & $\begin{array}{l}\begin{array}{l}\text { Human arm simulation for } \\
\text { interactive } \\
\text { environment design }\end{array} \\
\text { constrained }\end{array}$ & $\begin{array}{l}\text { International } \\
\text { Journal on } \\
\text { Interactive Design } \\
\text { and Manufacturing }\end{array}$ & $\mathrm{NE}$ & Scopus \\
\hline $\begin{array}{l}\text { HARIH; } \\
\text { ČRETNIK }\end{array}$ & 2013 & Eslovênia & $\begin{array}{l}\text { Interdisciplinary Approach to Tool- } \\
\text { Handle Design Based on Medical } \\
\text { Imaging }\end{array}$ & $\begin{array}{l}\text { BioMed Research } \\
\text { International }\end{array}$ & 2,476 & PubMed \\
\hline $\begin{array}{l}\text { OSTERLUND; } \\
\text { LAWRENCE }\end{array}$ & 2012 & $\begin{array}{l}\text { Estados } \\
\text { Unidos }\end{array}$ & $\begin{array}{l}\text { Virtual reality: Avatars in human } \\
\text { spaceflight training }\end{array}$ & Acta Astronautica & 1,536 & $\begin{array}{l}\text { Web of } \\
\text { Science/ } \\
\text { Scopus }\end{array}$ \\
\hline
\end{tabular}

Fonte: elaborado pelos autores. Legenda: NE= Não encontrado.

Com base na seleção do portfólio final de referências (teses e dissertações e bases de periódicos), convém destacar que os estudos são de origem dispersa como pode se observar no quadro 1 e 2. Vale destacar os países que mais estão utilizando os sistemas de captura de movimento para o desenvolvimento de produtos são a Coreia do Sul (5), os Estados Unidos (4), Reino Unido (2) e Brasil (2). Assim, na Coreia do Sul destacam-se: o Laboratório de Kookmin, o 
Departamento de Engenharia de Sistemas de Gestão da Sungkyunkwan University e o Departamento de Arquitetura da Hanyang University. Nos Estados Unidos a Buffalo University, Tufts University, Universidade de Oregon State, com o curso de Engenharia Mecânica e a Purdue University com os cursos de Engenharia Industrial e de Engenharia Agrícola e Biológica. No Reino Unido a Cranfield University e a Brunel University, com o curso de Engenharia Mecânica e o curso de Engenharia e Design. No Brasil, identificou-se a Universidade Federal de Santa Catarina, com o Centro Tecnológico do Programa de Pós- Graduação em Engenharia de Produção, o Núcleo de Gestão de Design e o Laboratório de Design e Usabilidade. Ainda foi identificada a Universidade Federal do Rio Grande do Sul, o curso de Engenharia e o Programa de Pós-Graduação em Design.

Salienta-se ainda que nos países como Eslovênia, China, Colômbia, Espanha, foram encontrados apenas uma publicação. Assim rastreou-se os laboratórios e departamentos de cada um desses países: Eslovênia (Laboratório de Sistemas Inteligentes da Faculdade de Engenharia Mecânica, o Departamento de Traumatologia do Centro Médico Universitário e o Departamento de Cirurgia da Faculdade de Medicina, todos da University of Maribor), China (Departamento de Engenharia Industrial da Tsinghua University), Colômbia (Universidade Militar de Nova Granada), Espanha (Departamento de Engenharia Mecânica do Centro de Estudos e Investigações Técnicas da Universidad Guipuzcoa). Essa análise permitiu identificar as Universidades, laboratórios e autores ao redor do mundo que estão trabalhando nesta área para futuras pesquisas acerca do tema.

Em relação as áreas do conhecimento científico, destacam-se as pesquisas em Engenharias, principalmente nas subáreas da Engenharia Mecânica e Industrial, como também as áreas de Arquitetura e Design. No que tange a evolução das publicações, percebe-se que o ano de 2014 foi o que mais teve publicações, sendo encontrado cinco publicações. Nos anos de 2017, 2015 e 2013 tiveram três publicações, enquanto em 2012 duas publicações e em 2016 apenas uma publicação.

Enfim, quanto aos periódicos analisados percebe-se que não há um periódico específico que contemple essa temática reunida, sendo que o periódico Sensors foi único que repetiu-se duas vezes na análise deste estudo. Em relação ao fator de impacto (JCR), referente aos periódicos selecionados, este se mostra alto, variando entre 0,628 e 3,997, por se tratar de uma área de estudo relativamente nova. Entretanto, salienta-se que o periódico Journal of Asian Architecture and Building Engineering, foi o único que apresentou um fator de impacto menor sendo de 0,277, conforme pôde ser observado no quadro 2.

\subsection{Análise do Conteúdo dos Documentos Selecionados}

No quadro 3, é apresentado os 17 documentos selecionados, sendo estes organizados por ano (do mais recente para o mais antigo). Nele é apresentado a abordagem/objetivo do estudo, bem como o tipo de equipamento tecnológico (captura de movimentos) utilizado.

Quadro 3 - Relação dos 17 documentos com identificação dos autores, ano, objetivo e equipamento utilizado

\begin{tabular}{lll}
\hline \multicolumn{1}{c}{ AUTOR/ ANO } & \multicolumn{1}{c}{ OBJETIVO } & \multicolumn{1}{c}{ EQUIPAMENTO } \\
\hline DEMIREL; & $\begin{array}{l}\text { Capturar atributos de ergonomia de usuários dentro do ciclo } \\
\text { virtual de desenvolvimento de produtos (carrinhos de } \\
\text { DUFFY, 2017 }\end{array}$ & $\begin{array}{l}\text { Sistema óptico } \\
\text { (STT Motion Captor) }\end{array}$ \\
\hline OYEKAN et al., 2017 & $\begin{array}{l}\text { Analisou a tarefa da montagem de um trilho do telhado de } \\
\text { um veículo. }\end{array}$ & $\begin{array}{l}\text { Sensor Kinect } \\
\text { (Captura de movimento em linha) }\end{array}$ \\
\hline
\end{tabular}




\begin{tabular}{|c|c|c|}
\hline LEE et al., 2017 & $\begin{array}{l}\text { Biomecânica no processo de projeto para desenvolvimento } \\
\text { de programas de treinamento e novas máquinas } \\
\text { (agachamento de Smith). }\end{array}$ & $\begin{array}{l}\text { Sistema Óptico } \\
\text { (Sistema de captura Vicon) }\end{array}$ \\
\hline $\begin{array}{l}\text { JOUNG; LI; } \\
\mathrm{NOH}, 2016\end{array}$ & $\begin{array}{l}\text { Propõe um método que automatiza a entrada de dados para } \\
\text { uma simulação ergonômica. }\end{array}$ & $\begin{array}{l}\text { Sensor Kinect } \\
\text { (Captura de movimento em linha) }\end{array}$ \\
\hline CHOI; LEE, 2015 & $\begin{array}{l}\text { Avaliação quantitativa do desconforto de entrada e saída de } \\
\text { caminhões com base em análises biomecânicas para o } \\
\text { movimento do corpo humano. }\end{array}$ & $\begin{array}{l}\text { Sistema Óptico } \\
\text { (Sistema de captura Vicon) }\end{array}$ \\
\hline JUN, 2015 & $\begin{array}{l}\text { Criação, análise e validação de um quadro de reabilitação. } \\
\text { Melhorar o processo de reabilitação para paciente de joelho } \\
\text { deficiente. }\end{array}$ & $\begin{array}{l}\text { Sistema Óptico (Sistema de captura } \\
\text { Vicon) e o Sistema Kinect (Captura } \\
\text { de movimento em linha) }\end{array}$ \\
\hline SOLOMON, 2015 & $\begin{array}{l}\text { Análise para investigar fatores alternativos da forma para a } \\
\text { serra sagital do osso com a intenção de reduzir a tensão do } \\
\text { operador, pressões localizadas e posturas de pulso } \\
\text { desviadas. }\end{array}$ & $\begin{array}{l}\text { Sensores eletromagnético } \\
\text { (Sensores, o G4 тм -Polhemus, } \\
\text { Colchester, VT) }\end{array}$ \\
\hline FERNANDES, 2014 & $\begin{array}{l}\text { Identificar os requisitos ergonômicos necessários ao projeto } \\
\text { de ferramentas manuais para a agricultura familiar, } \\
\text { especificamente para o caso da atividade de extração de } \\
\text { raízes de mandioca, com ênfase na Ergonomia física. }\end{array}$ & $\begin{array}{l}\text { Captura de movimento inercial } \\
\text { (XSENS) }\end{array}$ \\
\hline VELAZ et al., 2014 & $\begin{array}{l}\text { Apresenta a simulação de operações bimanuais de } \\
\text { montagem e desmontagem para treinamento ou aplicações } \\
\text { de design de produto. }\end{array}$ & Sis \\
\hline $\begin{array}{l}\text { ESPITIA- } \\
\text { CONTRERAS; } \\
\text { SANCHEZ-CAIMAN; } \\
\text { URIBE-QUEVEDO, } \\
2014\end{array}$ & $\begin{array}{l}\text { Desenvolvimento de uma ferramenta interativa para tomar } \\
\text { medidas antropométricas. }\end{array}$ & $\begin{array}{l}\text { Sensor Kinect } \\
\text { (Captura de movimento em linha) }\end{array}$ \\
\hline $\begin{array}{l}\text { JOUNG; } \\
\text { NOH, } 2014 \\
\end{array}$ & $\begin{array}{l}\text { Analise ergonômica de uma tarefa de montagem de } \\
\text { componentes de automóveis. }\end{array}$ & $\begin{array}{l}\text { Sensor Kinect } \\
\text { (Captura de movimento em linha) }\end{array}$ \\
\hline JI; JUN, 2014 & $\begin{array}{l}\text { Implementar um sistema de design generativo que possa } \\
\text { utilizar um algoritmo de design com uma tecnologia de } \\
\text { reconhecimento de movimento. }\end{array}$ & $\begin{array}{l}\text { Sensor Kinect } \\
\text { (Captura de movimento em linha) }\end{array}$ \\
\hline QING et al., 2013 & $\begin{array}{l}\text { Avaliar as diferenças do movimento humano para os } \\
\text { sujeitos sentados em uma cadeira de assento de Split } \\
\text { Ergokinetic com os sentados em cadeira de escritório } \\
\text { padrão. }\end{array}$ & $\begin{array}{l}\text { Sistema Óptico } \\
\text { (Software JACK) }\end{array}$ \\
\hline MA et al., 2013 & $\begin{array}{l}\text { Resolver parcialmente os problemas ergonômicos } \\
\text { encontrados durante a fase de concepção. }\end{array}$ & Sistema Óptico \\
\hline $\begin{array}{l}\text { HARIH; ČRETNIK, } \\
2013\end{array}$ & $\begin{array}{l}\text { Desenvolver um cabo da ferramenta com a forma ideal para } \\
\text { aumentar o desempenho, conforto, e a estabilidade na mão. }\end{array}$ & $\begin{array}{l}\text { Imaginologia por ressonância } \\
\text { magnética (MRI) e tomografia } \\
\text { computadorizada }\end{array}$ \\
\hline $\begin{array}{l}\text { OSTERLUND; } \\
\text { LAWRENCE, } 2012\end{array}$ & $\begin{array}{l}\text { Avaliar riscos ergonômicos dos técnicos de montagem no } \\
\text { desenvolvimento de veículo. }\end{array}$ & $\begin{array}{l}\text { Sistema Óptico } \\
\text { (Software JACK) }\end{array}$ \\
\hline SALVALAIO, 2012 & $\begin{array}{l}\text { Design de produtos assistivos, para o controle versátil de } \\
\text { cadeiras de rodas motorizadas a pessoas com deficiência } \\
\text { motora severa. }\end{array}$ & $\begin{array}{l}\text { Captura de movimento inercial } \\
\text { (XSENS); Flock of Birds; Luva 5DT }\end{array}$ \\
\hline
\end{tabular}

Fonte: elaborado pelos autores.

De acordo com o quadro 3, é possível perceber que os estudos concentram-se principalmente na área da Ergonomia, e salienta-se as análises ergonômicas para o desenvolvimento de produtos/sistemas/serviços (DEMIREL; DUFFY, 2017; LEE et al., 2017; JOUNG; LI; NOH, 2016; ESPITIA-CONTRERAS; SANCHEZ-CAIMAN; URIBE-QUEVEDO, 2014; JI; JUN, 2014; SALVALAIO, 2012), bem como no processo de concepção de novos produtos (JUN, 2015; SOLOMON, 
2015; FERNANDES, 2014; MA et al., 2013; HARIH; ČRETNIK, 2013), como também para a análise e avaliação de projetos (OYEKAN et al., 2017; CHOI; LEE, 2015; VELAZ et al., 2014; JOUNG; NOH, 2014; QING et al., 2013; OSTERLUND; LAWRENCE, 2012).

Neste sentido, os estudos que realizaram análises ergonômicas para o desenvolvimento de produtos estão relacionados a carinhos de limpeza (DEMIREL; DUFFY, 2017), equipamentos de ginástica, a citar, as máquinas de agachamentos (LEE et al., 2017), e o design de produto assistivo para controle de cadeiras de rodas motorizadas (SALVALAIO, 2012). No que refere-se aos sistemas, encontrou-se um método que automatiza a entrada dos dados para simulação ergonômica (JOUNG; $\mathrm{LI}$; NOH, 2016), bem como, o desenvolvimento de uma ferramenta interativa para mensurar medidas antropométricas (ESPITIA-CONTRERAS; SANCHEZ-CAIMAN; URIBE-QUEVEDO, 2014). No entanto o estudo de Ji e Jun (2014), buscaram desenvolver um sistema de design generativo que possa utilizar um algoritmo associado a uma tecnologia de captura de movimentos capaz de capturar os padrões comportamentais do usuário dentro do espaço físico.

No que tange a utilização da captura de movimentos para a concepção de novos produtos, o estudo de Solomon (2015), realizou análise biomecânica da mão e pulso, para melhorar a forma da serra sagital do osso, no intuito de reduzir a tensão do operador, pressões localizadas e posturas desviadas durante a tarefa. Neste mesmo sentido, o estudo de Harih e Cretnik (2013), buscou desenvolver um novo cabo para uma ferramenta cirúrgica com a finalidade de aumentar o desempenho, o conforto e a estabilidade nas cirurgias. Jun (2015) buscou melhorar a reabilitação de um paciente com problemas no joelho. E o estudo de Fernandes (2014) utilizou a captura de movimentos para propor requisitos ergonômicos de projeto para uma ferramenta manual de extração de mandioca. Ainda Velaz et al. (2014) propuseram análises de simulações de operações de montagem e desmontagem bimanuais para aplicações de treinamento ou design de produtos, visto que a maioria das aplicações de montagem têm sido limitadas para simular apenas tarefas unimanuais, ou tarefas bimanuais com uma mão.

Em relação a análise e avaliações de produtos, Qing et al (2013) realizaram uma análise biomecânica comparando as diferenças do movimento humano para sujeitos sentados em cadeira de assento de Split Ergokinetic com os sentados em cadeira de escritório. Ainda foram encontrados estudos ergonômicos, com a utilização da captura de movimentos na linha da indústria automotiva. A este respeito, Osterlund e Lawrence (2012) avaliaram os riscos ergonômicos que os técnicos de montagem estão sujeitos, quando realizam suas tarefas de desenvolvimento dos veículos. Já Joung e Noh (2014) analisaram os riscos da tarefa de montagem dos componentes de automóveis, e o estudo de Oyekan et al. (2017) avaliaram a tarefa da montagem do trilho do telhado de um veículo. Por fim, Choi e Lee (2015) analisaram o desconforto dos sujeitos ao entrar e sair de um caminhão.

Com base na identificação dos sistemas de captura de movimentos, destaca-se o sistema Óptico presente em 6 estudos (DEMIREL; DUFFY, 2017; LEE et al., 2017; CHOI; LEE, 2015; QING et al., 2013; MA et al., 2013; OSTERLUND; LAWRENCE, 2012;), o sistema Kinect, presente em 5 estudos (OYEKAN et al., 2017; JOUNG; LI; NOH, 2016; ESPITIA-CONTRERAS; SANCHEZ-CAIMAN; URIBEQUEVEDO, 2014; JOUNG; NOH, 2014; JI; JUN, 2014) e o sistema inercial Xsens, presente em 2 estudos (SALVALAIO, 2012 e FERNANDES, 2014). Ressalta-se que o estudo de (JUN, 2015), apresentou a utilização de dois sistemas de captura de movimento (o sistema Kinect, e o sistema óptico). Ainda, foram encontrados sensores eletromagnéticos no estudo de (SOLOMON, 2015), o sistema Markerless (VELAZ et al., 2014), o Flock of Birds e a Luva 5DT (SALVALAIO, 2012), como também a utilização da imaginologia por ressonância magnética associada a tomografia 
computadorizada (HARIH; CRETNIK, 2013).

\subsection{Síntese Visual dos Resultados}

Diante dos resultados apresentados, a figura 3, apresenta uma síntese visual da utilização da captura de movimentos no desenvolvimento de produtos, abordando os países de ocorrência, o número de publicações por ano, as áreas de concentração, as Instituições de Ensino Superior (IES), bem como os tipos de equipamentos utilizados e as aplicações dos estudos selecionados.

Figura 3 - Síntese visual dos resultados

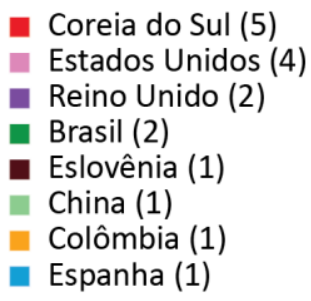

ANOS X NÚMERO DE PUBLICAÇÕES<smiles>[R20]C[R16]</smiles>

PRINCIPAIS ÁREAS DO CONHECIMENTO

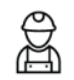

ENGENHARIA

Industrial Mecânica CIÊNCIAS APLICADAS

Arquitetura Design

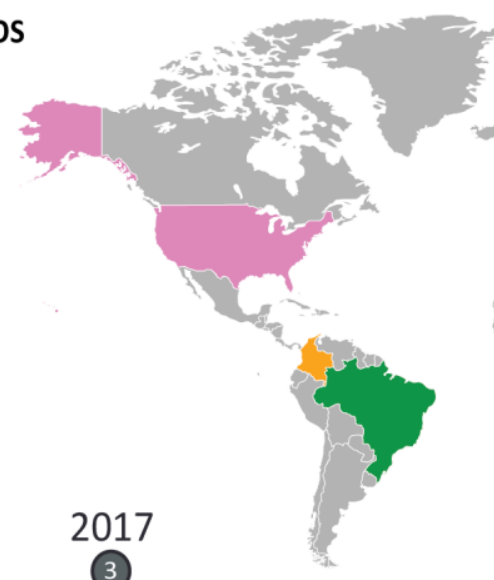

IES NACIONAIS
- UFSC
UFRGS

\section{IES INTERNACIONAIS}

- Sungkyunkwan University - Hanyang University

- Tufts University

- Oregon State University

- Purdue University

Buffalo University

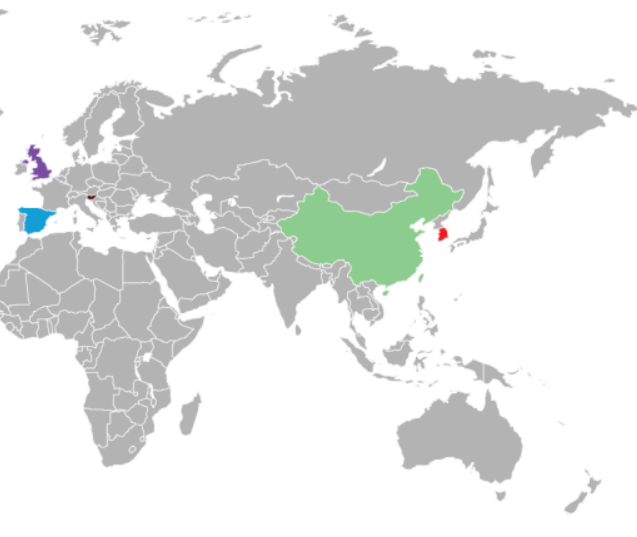

IES INTERNACIONAIS

Cranfield University

Brunel University - University of Maribor

Tsinghua University

- Universidad Guipuzcoa

- Universidade Militar Nova Granada

PRINCIPAIS SISTEMAS DE CAPTURA DE MOVIMENTOS

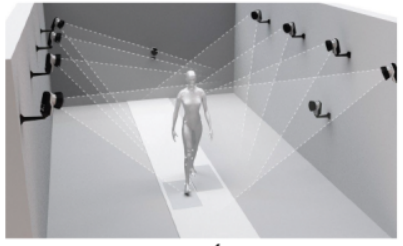

Sistema Óptico

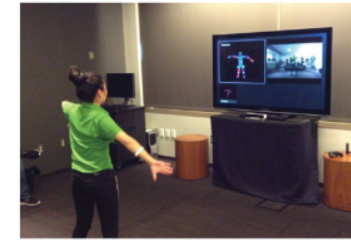

Sistema Kinect

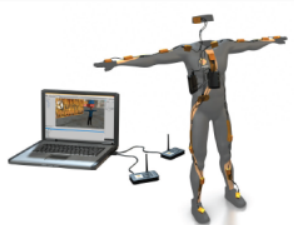

Sistema Inercial - Xsens

PRINCIPAIS APLICAÇÕES DA CAPTURA DE MOVIMENTOS

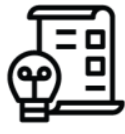

Desenvolvimento de produtos, sistemas e serviços

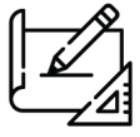

Processo de concepção de novos produtos

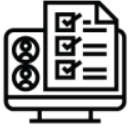

Análise e avaliação de projetos

Fonte: elaborado pelos autores. 


\section{Conclusão}

A ergonomia é uma área de conhecimento multidisciplinar que visa alcançar a melhor adequação entre o usuário, produto e contexto, em sua abrangência, principalmente a física, integrada aos conceitos da antropometria e biomecânica, com o intuito de estabelecer usabilidade, conforto e eficiência. Dessa forma, associar a instrumentação tecnológica, no desenvolvimento de projetos, permite aferições de dados precisos e confiáveis. Assim, mediante a necessidade de se desenvolver produtos adequados as capacidades e limitações dos usuários é que se buscou responder a seguinte questão: como a captura de movimentos vem sendo aplicada no desenvolvimento de produtos e/ou produtos de design e quais os tipos de equipamentos tecnológicos utilizados? Para isso, conduziu-se uma busca sistemática, com análise bibliométrica e de conteúdo dos 17 documentos selecionados, a fim de realizar um levantamento teórico sobre a utilização da captura de movimentos no desenvolvimento de produtos. Neste particular, os procedimentos adotados nesta pesquisa se mostraram satisfatórios para a obtenção dos resultados.

A partir da revisão foi possível identificar as tecnologias utilizadas na captura de movimentos e suas aplicações no desenvolvimento de produtos. Assim, nos documentos selecionados, os estudos utilizaram a captura de movimentos (Sistema Óptico, Sistema Kinect e Sistema Inercial) para aprimorar principalmente o processo de desenvolvimento de projetos, auxiliar na concepção de novos produtos e também contribuir para o processo de avaliação e análise dos projetos. A principal área do conhecimento no âmbito analisado é a Engenharia, na subárea da Mecânica e Industrial, seguida das áreas de Arquitetura e Design. Com relação ao país, destacam-se a Coreia do Sul, os Estados Unidos, Reino Unido e Brasil com maior número de publicações.

Os resultados dessa pesquisa, mostraram que a tecnologia da captura de movimentos tem sido utilizada tanto para análises com o usuário final (cliente) quanto para com o usuário indireto, ou seja, aquele que desenvolve o produto (mão de obra). Dessa forma, percebe-se que a captura de movimentos facilita o levantamento das capacidades e limitações dos usuários auxiliando na obtenção de medições objetivas (quantificáveis), gerando informações e definições precisas dos movimentos de segmentos e articulações, por meio de uma avaliação contínua do movimento, tornando-se um complemento positivo para as medidas subjetivas dos sujeitos.

Convém destacar também, que sua utilização possibilita a criação de novos produtos, guiando as etapas do processo e auxiliando na identificação dos requisitos de projetos. Ainda, pode contribuir na verificação e comparação do produto, garantindo assim maior viabilização do projeto. Assim, percebe-se que o equipamento de captura de movimentos pode ser uma ferramenta eficiente para a obtenção de dados quantitativos na área de design, gerando informações e definições precisas da biomecânica, aproximando o projetista as necessidades reais do usuário. Porém seu potencial parece ter sido pouco explorado, podendo ser explicado por aspectos como custos e disponibilidade do equipamento.

Como futuros estudos, sugere-se realizar um levantamento referente aos protocolos e métodos utilizados para a coleta de dados com os usuários, bem como compreender como os dados fornecidos pela instrumentação tecnológica são transformados em informações para auxiliar no desenvolvimento dos projetos. Ainda, salienta-se a importância de contemplar os demais domínios da ergonomia (cognitiva e organizacional), por serem aspectos fundamentais no desenvolvimento de produtos. Dessa forma, é possível dar início a estudos mais aprofundados, possibilitando resultados quantitativos e, portanto, mais precisos para as etapas de desenvolvimento de produtos. 


\section{Referências}

BDTD. BDTD 15 anos. Biblioteca Digital de Teses e Dissertações. In: BDTD, 2018. (http://bdtd.ibict.br/vufind/).

BEST, Kathryn. Design Management: managing design strategy, process and implementation. Nova York: Bloomsbury, 2015.

BITTENCOURT, Fábio. Antropometria: Conceitos. In Fábio Bittencourt (Org.) Ergonomia e Conforto Humano: Uma visão da arquitetura, engenharia e design de interiores. 1 ed. Rio de Janeiro: Rio Books, 2011.

BONSIEPE, Gui. Design: como prática de projeto. São Paulo: Blucher, 2012.

CAMBIAGHI, Silvana. Desenho Universal: métodos e técnicas para arquitetos e urbanistas. São Paulo: Senac, 2012.

CARDOSO, Rafael Denis. Design para um mundo complexo. São Paulo: Cosac Naify, 2012.

CATÁLAGO DE TESES E DISSERTAÇÕES. In: Catálogo de teses e dissertações, 2018. (http://catalogodeteses.capes.gov.br/catalogo-teses/\#!/)

CHOI, Nam-Chul; LEE, Sang Hun. Discomfort evaluation of truck ingress/egress motions based on biomechanical analysis. Sensors, v. 15, n. 6, p. 13568-13590, 2015.

CRESWELL, John W. Projeto de pesquisa: Métodos qualitativo, quantitativo e misto. 3 ed. Porto Alegre: Artmed, 2014.

DEMIREL, Onan H.; DUFFY, Vincent G. Incorporating Tactile Cues into Human-Centered Virtual Product Design. Human Factors and Ergonomics in Manufacturing \& Service Industries, v. 27, n. 1, p. 5-16, 2017.

ESPITIA-CONTRERAS, Alvaro; SANCHEZ-CAIMAN, Pedro; URIBE-QUEVEDO, Alvaro. Development of a Kinect-based anthropometric measurement application. In: Virtual Reality (VR), 2014 IEEE. IEEE, 2014. p. 71-72.

FERNANDES, Carlos Aparecido. ERGONOMIA E PROJETO: contribuições no projeto de ferramentas manuais para agricultura familiar com ênfase nos reflexos físicos da atividade de extração manual de mandioca. 2014. Tese (Doutorado)- Curso de Pós-graduação em Engenharia de Produção, Universidade Federal de Santa Catarina, Florianópolis, 2014. Disponível em:<https://repositorio.ufsc.br/bitstream/handle/123456789/123438/325946.pdf?sequence=1 $>$. Acesso em: 23 set.2017.

HAIR JR, Joseph F. et al. Fundamentos em métodos de pesquisa em administração. Porto Alegre: Bookman, 2005.

HARIH, Gregor; ČRETNIK, Andrej. Interdisciplinary approach to tool-handle design based on medical imaging. BioMed research international, v. 2013, 2013.

INTERNATIONAL ERGONOMICS ASSOCIATION (IEA). Definition and Domains of Ergonomics. 2017. Disponível em:< http://www.iea.cc/whats/index.html> Acesso em: 30 out.2017.

JI, Seung Yeul; JUN, Han Jong. Elementary Implementation of a Parameter-Based Generative Design System Considering the User Environment. Journal of Asian Architecture and Building Engineering, v. 13, n. 2, p. 333-340, 2014. 
JOUNG, Youn-Kyoung; LI, Quanri; NOH, Sang Do. XML-based neutral schema for automated ergonomic analysis with digital human simulation and inline motion capture. International Journal of Computer Integrated Manufacturing, v. 29, n. 1, p. 25-41, 2016.

JOUNG, Youn-Kyoung; NOH, Sang Do. Integrated modeling and simulation with in-line motion captures for automated ergonomic analysis in product lifecycle management. Concurrent Engineering, v. 22, n. 3, p. 218-233, 2014.

JUN, Seungkook. A home-based rehabilitation system for deficient knee patients. 2015. Tese (Doutorado)- Curso de Pós graduação em Engenharia Mecânica e Aeroespacial, State University of New York at Buffalo, Buffalo, 2015. Disponível em:< https://search.proquest.com/docview/1709470204?pq-origsite=gscholar> Acesso em: 18 nov.2015.

LEE, Haerin et al. A 3D Human-Machine Integrated Design and Analysis Framework for Squat Exercises with a Smith Machine. Sensors, v. 17, n. 2, p. 299, 2017.

MA, Liang et al. Human arm simulation for interactive constrained environment design. International Journal on Interactive Design and Manufacturing, p. 1-10, 2013.

MARCONI, Marina de Andrade; LAKATOS, Eva Maria. Técnicas de pesquisa: planejamento e execução de pesquisas, amostragens e técnicas de pesquisas, elaboração, análise e interpretação de dados. 6.ed. São Paulo: Atlas, 2007.

MARTINS, Rosane Fonseca D. F.; MERINO, Eugenio A. D. Gestão de design como estratégia organizacional. Rio de janeiro: Rio Books, 2011.

MERINO, Eugenio Andrés Díaz et al. Instrumentação tecnológica integrada no desenvolvimento de produtos ergonômicos para Tecnologia Assistiva. Anais de congresso. 1을 Congresso Brasileiro de Pesquisa e Desenvolvimento em Tecnologia Assistiva (CBTA), Curitiba/PR, Brasil, 2016a.

. GODP - metodologia de projeto centrado no usuário: multicasos de projetos de Tecnologia Assistiva na Terapia Ocupacional. Anais de congresso. 1을 Congresso Brasileiro de Pesquisa e Desenvolvimento em Tecnologia Assistiva (CBTA), Curitiba/PR, Brasil, 2016b.

NEUMANN, Donald A. Cinesiologia do aparelho musculoesquelético: fundamentos para reabilitação. 2. ed. Rio de Janeiro: Elsevier, 2011.

OLIVEIRA, Márlis Abreu Marques. Biomecânica. In Fábio Bittencourt (Org.) Ergonomia e Conforto Humano: Uma visão da arquitetura, engenharia e design de interiores. 1 ed. Rio de Janeiro: Rio Books, 2011.

OSTERLUND, Jeffrey; LAWRENCE, Brad. Virtual reality: Avatars in human spaceflight training. Acta Astronautica, v. 71, p. 139-150, 2012.

OYEKAN, John et al. Remote real-time collaboration through synchronous exchange of digitised human-workpiece interactions. Future Generation Computer Systems, v. 67, p. 83-93, 2017.

PROQUEST. ProQuest Dissertations \& Theses Global. In: ProQuest, 2018.

(https://search.proquest.com/pqdtglobal/index?accountid=26642).

PUBMED. PubMed. gov. In: NCBI, 2018. (https://www.ncbi.nlm.nih.gov/pubmed/).

QING, Tao et al. Application of JACK on evaluation of a split seat chair. In: Automation and 
Computing (ICAC), 2013 19th International Conference on. IEEE, p.1-6, 2013.

RI UFSC. Repositório Institucional UFSC. In: RI UFSC, 2018. (https://repositorio.ufsc.br/).

SALVALAIO, Cláudio Luiz. Contribuição ao estudo da captura do movimento aplicado ao design em tecnologia assistiva. 2012. 122 f. Dissertação (Mestrado) - Curso de Pós-design, Engenharia de Materiais, Universidade Federal do Rio Grande do Sul, Porto Alegre, 2012. Disponível em: <http://www.lume.ufrgs.br/handle/10183/62049>. Acesso em: 06 out. 2017.

SAMPAIO, Rosana F.; MANCINI, Marisa C. Systematic review studies: a guide for careful synthesis of the scientific evidence. Brazilian Journal of Physical Therapy, v. 11, n. 1, p. 83-89, 2007.

SAMPIERI, Roberto Hernández; COLLADO, Carlos Fernández; LUCIO, María del Pilar Baptista. Metodologia de pesquisa. 5.ed. Porto Alegre: Penso, 2013.

SCIELO. Scielo 20 anos. In: Scielo, 2018. (http://www.scielo.org).

SCOPUS. Document search. In: SCOPUS, $2018 . \quad$ (https://wwwscopus.ez46.periodicos.capes.gov.br/search/form.uri?display=basic).

SOLOMON, Tabitha A. Application of human factors engineering in the redesign of an oscillating bone saw to reduce high-risk postures of the wrist and to enhance performance. 2015. Tese (Doutorado) - Curso de Pós-graduação em Engenharia dos Fatores Humanos, Tufts University, Medford, 2015. Disponível em: < https://dl.tufts.edu/catalog/tufts:21546> Acesso em: 18 nov.2017.

VELAZ, Yaiza et al. Natural and hybrid bimanual interaction for virtual assembly tasks. Virtual Reality, v. 18, n. 3, p. 161-171, 2014.

WEB OF SCIENCE. Pesquisa. In: Web of Science, 2018. (http://appswebofknowledge.ez46.periodicos.capes.gov.br/SCIELO_GeneralSearch_input.do?product=SCIELO \&search_mode=GeneralSearch\&SID=8BzIXjBfzekIfSYoeBf\&preferencesSaved).

WDO (World Design Organization). In: Definition of Industrial Design, 2017. (http://wdo.org/about/definition/).

\section{Agradecimentos}

Agradecemos ao Programa de Pós-graduação em Design da UFSC (PPGD/UFSC), ao Núcleo de Gestão de Design e Laboratório de Design e Usabilidade (NGD-LDU/UFSC), à Rede de Pesquisa e Desenvolvimento em Tecnologia Assistiva (RPDTA), à Coordenação de Aperfeiçoamento de Pessoal de Nível Superior (CAPES), ao Conselho Nacional de Desenvolvimento Científico e Tecnológico (CNPq), e aos demais envolvidos. 